\title{
Bone mineral density in systemic lupus erythematosus: comparison with rheumatoid arthritis and healthy controls
}

\author{
Inge-Margrethe Gilboe, Tore K Kvien, Glenn Haugeberg, Gunnar Husby
}

\begin{abstract}
Objectives-To examine bone mineral density (BMD) frequency of osteoporosis and reduced bone mass in systemic lupus erythematosus (SLE), and compare the data of the SLE patients with matched rheumatoid arthritis (RA) patients and healthy controls. Secondly, to study possible correlations between BMD, demographic and disease variables in the SLE patients.
\end{abstract}

Methods-Measures of BMD assessed by dual energy $x$ ray absorptiometry were obtained from 75 SLE patients aged $\leqslant 70$ years, 75 RA patients matched for age, sex and disease duration, and from 75 healthy controls matched for age, sex and geographical area. Disease activity and accumulated organ damage were assessed in the SLE patients.

Results-The SLE patients had significantly lower BMD values at lumbar spine L2-L4 and hip, and higher frequency of osteoporosis at all sites of measurement compared with matched healthy controls. The matched SLE and RA patients had similar BMD, prevalence of osteoporosis and reduced bone mass. In the SLE patients BMD was more strongly correlated with accumulated organ damage than with markers of disease activity or duration. In multivariate analyses BMD was at all sites predicted by age and body mass, at lumbar spine also by the current corticosteroid dose.

Conclusion-The study showed reduced BMD in patients with SLE compared with matched healthy controls. Premenopausal women taking corticosteroids were especially affected. Furthermore, the BMD of matched SLE and RA patients was reduced to a similar extent.

(Ann Rheum Dis 2000;59:110-115)

of Rheumatology,

Hospital, Box 23

Vinderen, N-0319 Oslo,

Norway

I-M Gilboe

T K Kvien

G Haugeberg

Centre for Rheumatic Diseases, The National Hospital, Oslo, Norway G Husby

Correspondence to: Dr Gilboe

Accepted for publication 4 November 1999 fragility, and consequently increased risk of fractures may be related to the underlying dis-
Improved survival of systemic lupus erythemaput morbidity as outcome measure into focus. for the disease.

$\mathrm{OP}$, characterised by a reduction in bone mineral density (BMD), microarchitectural ease process in SLE, its treatment or the risk factors in the general population. ${ }^{2}$

Although a large body of data on OP in RA has emerged, literature investigating $\mathrm{OP}$ in SLE is practically non-existing, and many previous studies of OP in SLE have used older methods, making comparison to newer studies difficult. Dual energy $x$ ray absorptiometry (DEXA) has been used in some studies in SLE. ${ }^{3-11}$ The majority of these have been limited to small samples of premenopausal women. ${ }^{3-5} 5^{7-9} 11$ BMD in SLE has previously been compared with healthy controls ${ }^{35-9}$ and with RA. ${ }^{12-14}$ However, none of the studies comparing SLE and RA $^{12-14}$ have used DEXA. Moreover, examination of BMD measured by DEXA has not been applied in studies comparing SLE, RA and healthy controls at the same time.

The main objective of this study was therefore to examine BMD in SLE patients, using DEXA, and to compare BMD in SLE patients with two control groups, one comprising by age and sex matched healthy controls and one by age, sex and disease duration matched patients with rheumatoid arthritis (RA). We further intended to study the relations between BMD, demographic and disease variables in the SLE patients.

\section{Methods}

SETTING

The study was performed at Diakonhjemmet hospital, Oslo City Department of Rheumatology, which offers rheumatological service for the community of Oslo, the capital city of Norway with approximately 500000 inhabitants. Previous studies have demonstrated that the community of Oslo serves as a reliable setting for the performance of epidemiological studies in rheumatology..$^{15} 16$

PATIENTS AND HEALTHY CONTROLS A cohort of 93 SLE patients fulfilling the revised classification criteria for SLE, ${ }^{17}$ and with residential address in Oslo, has been followed up longitudinally since 1995/1996. Eighty seven of them participated in a follow up examination in 1997-98, of whom patients $\leqslant 70$ years $(n=80)$ were eligible for this study. Five refused to participate, leaving 75 patients for inclusion in the study. The five nonparticipants did not differ from the participants regarding demographic and disease variables.

Seventy five RA patients ${ }^{18}$ matched to the SLE patients for sex, age and disease duration ( \pm 2 years) were recruited from the Oslo RA 
register at Diakonhjemmet Hospital. The register was established in 1991, comprising patients with RA with disease onset after the age of 16 years and with a residential address in Oslo. ${ }^{15}$

People used as healthy controls were selected from the Population register of Oslo. Each control was matched to one of the SLE patients with regard to age, sex and geographical area of Oslo, consistent with a case-control design of the study. The healthy controls received an invitation by mail, and the initial nonrespondents received a reminder after two weeks. None of the healthy controls had rheumatic disease or used corticosteroids for other health conditions.

DATA COLLECTION AND CLINICAL MEASURES

The SLE, RA patients and healthy controls consenting to participate were examined at the outpatient clinic by a standardised interview and BMD. The assessments of the SLE patients were performed in 1997-1998, and RA patients in 1996-97 and the healthy controls in 1998.

Data collected from the interview included menstrual status, age at and duration of menopause, smoking habits and previous osteoporotic fractures. Data on physical disability were collected by self reported questionnaires (Modified Health Assessment Questionnaire $(\mathrm{MHAQ})^{19}$ and SF-36 ${ }^{20}$ filled in the day before or during the visit. MHAQ is a modified shortened version of the Stanford Health Assessment Questionnaire, ${ }^{21}$ examining eight dimensions of difficulties with the performance of activities of daily living, scaled 1-4. SF-36 physical is the physical part of the generic instrument, MOS Short Form SF-36, measuring eight dimensions of health status. ${ }^{20}$ The scales of SF-36 are expressed with values from 0 to 100 , with higher value representing better functioning/health. The version covering the past four weeks was used.

The SLE and RA patients underwent a clinical examination and a careful review of their medical records, performed by one of us (IMG) for the SLE patients and by a trained research nurse in collaboration with a rheumatologist for the RA patients. Number of tender and swollen joints was assessed by the 28 joint count in both patient groups.

Disease activity in the SLE patients was assessed by the SLE disease activity index (SLEDAI), ${ }^{22}$ and accumulated organ damage by the Systemic Lupus International Collaborating Clinics/ American College of Rheumatology Damage index (SDI). ${ }^{1}$ The SLEDAI is a validated disease activity measure index. The version covering the past 10 days was used. It contains 24 descriptors in nine organ systems, including clinical and laboratory measures of SLE activity, and is weighted to reflect the degree of activity. The maximum possible score is 105 . The SDI is designed to assess accumulated organ damage in the SLE since onset of the disease, caused by the disease itself or the treatment. Damage is defined for 12 organ systems and the score can only increase over time, theoretically to a maximum of 47 .
Disease onset was defined as the time when the patients fulfilled the ARA/ACR criteria for the respective diseases (SLE and RA); disease duration as the period from the disease onset to the time of this study.

BONE MINERAL DENSITY MEASUREMENT

BMD of the lumbar spine (L2-L4) and the left hip (femoral neck and total hip) was measured by DEXA equipment (Lunar Expert, Madison, Wisconcin), and expressed in $\mathrm{g} / \mathrm{cm}^{2}$. The machine was calibrated daily with a spine phantom provided by the manufacturer. The measurement was assessed according to standard procedures. The spine was investigated with the patients in a supine position, straightening the lordotic curvature by elevating the legs with help of a squared pillow (hip and knee flexion 90 degrees). Measurements of the hip were performed with the patient's left foot in a fixture to position the femoral neck parallel to the table. In two RA patients, the right hip was measured, because of prosthesis in the left.

The spine phantom precision error calculated as a coefficient of variation $(\mathrm{CV} \%)$ was $0.9 \%$ for the whole period (1996 to 1998 ). The in vivo reproducibility of BMD measurements was assessed from duplicate measurements in 31 healthy female hospital workers (mean age 56.1 years, range 50-66). The precision was $2.5 \%$ at lumbar spine $\mathrm{L} 2-\mathrm{L} 4,1.5 \%$ at total hip and femoral neck. The inter-observer precision variation was $0.7-1.4 \%$ for spine $\mathrm{L} 2-\mathrm{L} 4$, $0.4-0.5 \%$ for the total hip and $0.5-0.8 \%$ for the femoral neck.

DATA ANALYSES AND STATISTICS

The BMD in SLE was compared with BMD of matched RA and matched healthy controls. $\mathrm{BMD}$ was also expressed as $\mathrm{T}$ score (number of standard deviations (SD) from the mean of young women attaining peak bone mass) and $\mathrm{Z}$ score (number of standard deviations of age matched controls adjusted for weight), using the normative German reference values provided by the manufacturer. Osteoporosis was defined according to the conventional WHO definition $\mathrm{T}$ score $<-2.5 \mathrm{SD},{ }^{23}$ and reduced bone mass as $\mathrm{T}$ score and $\mathrm{Z}$ score below -1 SD.

All statistical analyses were performed by SPSS version 8.0. Descriptive statistics are presented as means with range, standard deviation (SD) or $95 \%$ confidence intervals (CI). Group comparisons were analysed by one way variance (ANOVA) with Bonferroni corrections. $\mathrm{F}$ ratios for group comparisons and overall $\mathrm{p}$ are presented. $t$ Tests for independent samples were used to compare the means of two groups. Categorical variables were compared by $\chi^{2}$ test. The differences were regarded as statistically significant when $p<0.05$ and highly significant when $\mathrm{p}<0.01$. Bivariate relations were examined by Pearson's correlation coefficients and $t$ tests. A correlation between continuous variables was considered as strong if the correlation coefficient was 0.70 or higher, moderate to substantial if between $0.30-0.70$, and weak if below 0.30 . Predictors of BMD in SLE was first examined by bivariate 
Table 1 Descriptive characteristics of the study populations. (Mean (range) for continuous, \% for categorical variables)

\begin{tabular}{llll}
\hline & $S L E(n=75)$ & $R A(n=75)$ & Controls $(n=75)$ \\
\hline Women & 88 & 88 & 88 \\
Age $(y)$ & $45(20-70)$ & $45(22-70)$ & $45(20-70)$ \\
White & 100 & 100 & 100 \\
Disease duration (y) & $8.1(2-27)$ & $7.9(2-26)$ & NA \\
Body mass index & $23.5(17.7-37.6)$ & $23.4(16.6-36.5)$ & $24.0(18.7-36.3)$ \\
Smokers (current or previous) & 68 & 63 & 56 \\
Menopause & 43 & 32 & 31 \\
Oestrogen replacement therapy & & & \\
Ever users & 24 & 24 & 20 \\
Current users & 19 & 19 & 19 \\
Osteoporotic fractures & 11 & 3 & 4 \\
MHAQ & $1.32(1.0-3.00)^{\star}$ & $1.53(1.0-2.75)$ & $1.07(1.0-1.88)^{\star \star}$ \\
SF-36 physical & $66.8(5-100)^{\star}$ & $57.5(0-100)$ & $89.7(20-100)^{\star \star}$ \\
SLEDAI & $6.93(0-24)$ & $\mathrm{NA}$ & $\mathrm{NA}$ \\
SDI & $2.07(0-13)$ & $\mathrm{NA}$ & $\mathrm{NA}$ \\
Tender joints & $3.2(0-28)^{\star}$ & $6.1(0-23)$ & $\mathrm{NA}$ \\
Swollen joints & $2.0(0-21)^{\star}$ & $7.3(0-23)$ & $\mathrm{NA}$ \\
Corticosteroids & & & \\
$\quad$ Ever users & $85^{\star}$ & 64 & 0 \\
Current users & 60 & 45 & 0 \\
Mean current dose & $7.4(2.5-20)$ & $6.6(2.5-20)$ & NA \\
Cumulative dose $(\mathrm{g})$ & $21.8(0.23-113.4)^{\star}$ & $11.8(0-75.6)$ & NA \\
Duration, months & $72.6(1-364)$ & $52.2(0-336)$ & NA \\
Current NSAIDs & $17^{\star}$ & 44 & NA \\
Current DMARDs & 35 & 32 & NA \\
Current cytotoxic drugs & 19 & 29 & NA \\
\hline * & & &
\end{tabular}

${ }^{\star} \mathrm{p}<0.05$ SLE $v$ RA. ${ }^{\star \star} \mathrm{p}<0.001$ SLE and RA $v$ healthy controls. NA : not applicable, MHAQ : Modified Health Assessment Questionnaire, SLEDAI : SLE Disease Activity Index, SDI : Systemic Lupus International Collaborating Clinics/American College of Rheumatology Damage Index, NSAID : non-steroidal anti-inflammatory drug, DMARD : disease modifying antirheumatic drug.

statistical tests and then by multivariate linear regression analyses.

ETHICS AND LEGAL ASPECTS

The local ethical committee had approved the study and the Data Inspectorate the register of SLE and RA patients in Oslo.

\section{Results}

PATIENTS

As shown in table 1 the study populations comprised 75 SLE patients, 75 matched RA patients and 75 matched healthy controls. Eighty eight per cent of the patients and controls were women. The matched SLE, RA patients and healthy controls were all white and did not differ significantly with respect to body mass index (BMI) and smoking habits. A higher proportion of the SLE patients was menopausal, but the difference between the groups did not reach statistical significance. The use of oestrogen replacement therapy was similar in the three groups. The proportions of patients and healthy controls reporting osteoporotic fractures including Colles fracture were somewhat higher in the SLE group. Number of tender and swollen joints as well as scores of physical disability were, as expected, significantly different in SLE and RA, in the direction of poorer health in RA. Both patient groups were significantly more physically disabled than healthy controls.
The proportion of SLE patients using or having used corticosteroids and the cumulative corticosteroid dose was significantly higher in SLE compared with RA patients, and the duration of corticosteroid treatment somewhat longer in SLE. As expected, the RA patients used significantly more non-steroidal antiinflammatory drugs (NSAIDs). The use of disease modifying antirheumatic drugs (DMARDs) and cytotoxic drugs was similar in the two groups (table 1).

COMPARISON OF SLE PATIENTS AND MATCHED HEALTHY CONTROLS

Table 2 displays BMD values for SLE, matched RA patients and healthy controls. The $F$ ratios revealed substantial differences between the three groups.

The SLE patients had significantly lower BMD values at lumbar spine L2-L4 (trabecular bone), at femoral neck and at total hip (mixed cortical and trabecular bone) compared with matched healthy controls (table 2). The bone mass reduction was $7 \%$ at all sites of measurement compared with healthy controls. Subgroup analyses showed that the premenopausal SLE women had significantly lower BMD than matched controls, whereas the postmenopausal patients and the patients having never received corticosteroids had BMD levels approaching the healthy controls (data not shown).

The frequency of SLE patients with osteoporosis ( $\mathrm{T}$ scores $<-2.5 \mathrm{SD}$ ) was overall higher compared with healthy controls, but the differences did not reach statistical difference ( $9 \%$ versus $4 \%$ at lumbar spine, $7 \%$ versus $3 \%$ at femoral neck and $4 \%$ versus $0 \%$ at total hip, SLE versus healthy controls, respectively). The proportion of SLE patients having reduced bone mass ( $T$ score $<-1 \mathrm{SD}$ ) was significantly higher at femoral neck (41\% versus $22 \%$ ) compared with healthy controls, whereas the increased proportions were not significantly increased at lumbar spine $(37 \%$ versus $28 \%)$, at total hip (33\% versus $20 \%$ ) SLE versus healthy controls, respectively. The proportion of SLE patients having Z score $<-1$ SD was higher than the expected $16 \%$ (because of the normal distribution) at all sites and ranged from $21 \%$ at the total hip to $29 \%$ at lumbar spine, significantly increased at lumbar spine. The healthy controls ranged from $12 \%$ to $15 \%$, comparing well with the normal distribution (data not shown).

\section{COMPARISON OF SLE AND RA PATIENTS}

The SLE patients had similar BMD values at lumbar spine L2-L4, at femoral neck and total hip, compared with sex, age and disease duration matched RA patients (table 2).

Table 2 Mean BMD values with 95\% confidence intervals in SLE, matched RA patients and healthy controls

\begin{tabular}{|c|c|c|c|c|c|c|c|c|}
\hline & \multicolumn{2}{|l|}{$S L E$} & \multicolumn{2}{|l|}{$R A$} & \multicolumn{2}{|c|}{ Healthy controls } & \multirow[b]{2}{*}{$F$ ratiof } & \multirow[b]{2}{*}{$p$ valuet } \\
\hline & $(n=75)$ & $(95 \% C I)$ & $(n=75)$ & $(95 \% C I)$ & $(n=75)$ & $(95 \% C I)$ & & \\
\hline Lumbar spine L2-L4 & $1.12^{\star \star}$ & $1.08,1.16$ & 1.17 & $1.12,1.22$ & 1.21 & $1.17,1.25$ & 4.527 & 0.01 \\
\hline Femoral neck & $0.92^{\star}$ & $0.88,0.95$ & $0.92^{\star}$ & $0.88,0.96$ & 0.98 & $0.95,1.02$ & 3.778 & 0.02 \\
\hline Total hip & $0.94^{\star}$ & $0.91,0.98$ & $0.94^{\star}$ & $0.90,0.98$ & 1.01 & $0.98,1.05$ & 4.960 & 0.01 \\
\hline
\end{tabular}

${ }^{\star} \mathrm{p}<0.05 \mathrm{SLE}$ and/or RA $v$ controls (Bonferroni). ${ }^{\star \star} \mathrm{p}<0.01 \mathrm{SLE}$ and/or RA $v$ controls (Bonferroni). †ANOVA. 
Table 3 Bivariate relations of BMD at lumbar spine and femoral neck and demographic and disease variables in 75 SLE patients: Pearson's correlation coefficient $r$ for continuous variables and t test for group comparison of $B M D\left(\mathrm{~g} / \mathrm{cm}^{2}\right)($ mean $(S D))$

\begin{tabular}{|c|c|c|c|c|}
\hline & \multicolumn{2}{|c|}{ Lumbar spine L2-L4 } & \multicolumn{2}{|l|}{ Femoral neck } \\
\hline & $r$ & $p$ & $r$ & $p$ \\
\hline Age & -0.25 & 0.03 & -0.34 & $<0.01$ \\
\hline BMI & 0.32 & 0.01 & 0.29 & 0.01 \\
\hline Disease duration & -0.15 & 0.21 & -0.03 & 0.83 \\
\hline Current corticosteroid dose & -0.27 & 0.02 & -0.21 & 0.08 \\
\hline Cumulative corticosteroid dose & -0.22 & 0.06 & -0.20 & 0.09 \\
\hline Total corticosteroid duration & -0.13 & 0.26 & -0.15 & 0.19 \\
\hline SLEDAI & 0.07 & 0.57 & 0.02 & 0.86 \\
\hline \multirow[t]{2}{*}{ SDI } & -0.26 & 0.02 & -0.33 & $<0.01$ \\
\hline & $B M D$ & $p$ & $B M D$ & $p$ \\
\hline \multicolumn{5}{|l|}{ Corticosteroid use } \\
\hline Current users & $1.093(0.16)$ & 0.04 & $0.898(0.15)$ & 0.16 \\
\hline Current non-users & $1.166(0.15)$ & & $0.950(0.16)$ & \\
\hline Ever users & $1.118(0.15)$ & 0.61 & $0.917(0.16)$ & 0.77 \\
\hline Never users & $1.145(0.20)$ & & $0.931(0.16)$ & \\
\hline \multicolumn{5}{|l|}{ Postmenopausal } \\
\hline Yes & $1.073(0.17)$ & 0.02 & $0.867(0.14)$ & 0.01 \\
\hline No & $1.158(0.14)$ & & $0.958(0.16)$ & \\
\hline \multicolumn{5}{|l|}{ Sex } \\
\hline Female & $1.115(0.16)$ & 0.31 & $0.905(0.15)$ & 0.04 \\
\hline Male & $1.173(0.14)$ & & $1.022(0.17)$ & \\
\hline \multicolumn{5}{|l|}{ Smoker (ever) } \\
\hline Yes & $1.134(0.17)$ & 0.31 & $0.925(0.16)$ & 0.65 \\
\hline No & $1.094(0.16)$ & & $0.907(0.15)$ & \\
\hline
\end{tabular}

BMI : body mass index. Other abbreviations as in table 1.

Table 4 Multiple regression analyses of BMD at lumbar spine and femoral neck (dependent variables), demographic and disease variables (independent variables) in 75 SLE patients

\begin{tabular}{|c|c|c|c|c|c|c|c|c|}
\hline \multirow{3}{*}{$\begin{array}{l}\text { Predictors } \\
\text { Age }\end{array}$} & \multicolumn{4}{|c|}{ Lumbar spine L2-L4 } & \multicolumn{4}{|c|}{ Femoral neck } \\
\hline & \multicolumn{2}{|c|}{$\begin{array}{l}\text { Adjusted } \\
\text { estimate } \beta^{\star}\end{array}$} & \multirow{2}{*}{$\frac{S E t}{0.001}$} & \multirow{2}{*}{$\begin{array}{l}p \\
0.01\end{array}$} & \multicolumn{2}{|c|}{$\begin{array}{l}\text { Adjusted } \\
\text { estimate } \beta^{\star}\end{array}$} & \multirow{2}{*}{$\begin{array}{l}\text { SEt } \\
0.001\end{array}$} & \multirow{2}{*}{$\begin{array}{l}p \\
<0.01\end{array}$} \\
\hline & -3.8 & $10^{-3}$ & & & -4.4 & $10^{-3}$ & & \\
\hline Sex & -2.2 & $10^{-2}$ & 0.510 & 0.66 & -8.8 & $10^{-2}$ & 0.049 & 0.07 \\
\hline BMI & 1.3 & $10^{-2}$ & 0.004 & $<0.01$ & 1.1 & $10^{-2}$ & 0.004 & 0.01 \\
\hline Current corticosteroid dose & -8.1 & $10^{-3}$ & 0.004 & 0.03 & -5.3 & $10^{-3}$ & 0.003 & 0.13 \\
\hline Cumulative corticosteroid dose & -2.2 & $10^{-7}$ & 0.000 & 0.16 & -1.6 & $10^{-7}$ & 0.000 & 0.29 \\
\hline SDI & -9.8 & $10^{-4}$ & 0.009 & 0.92 & -5.8 & $10^{-3}$ & 0.009 & 0.53 \\
\hline$r^{2}(\%) \ddagger$ & & & & 22 & & & & 27 \\
\hline
\end{tabular}

*Adjusted estimate $\beta$ : The regression coeffient, when considered in the multiple regression model. †SE : standard error of $\beta . \ddagger r^{2}$ : total explained variance of the model.

Furthermore, no significant differences in BMD appeared when comparing subgroups stratified for their menopausal status (data no shown). Furthermore, the overall frequencies of OP and reduced bone mass were similar. In SLE, the frequency of OP ranged from $4 \%$ at total hip to $9 \%$ at lumbar spine, in RA from $4 \%$ at total hip and femoral neck to $5 \%$ at lumbar spine (data not shown).

Reduced bone mass ( $\mathrm{T}$ score $<-1 \mathrm{SD}$ ) ranged from $33 \%$ to $41 \%$ in SLE and $28 \%$ to $44 \%$ in RA at different sites, whereas the proportion of SLE and RA patients with Z score value $<-1$ SD was higher than the expected $16 \%$, and ranged from $21 \%$ to $29 \%$ in SLE and from $16 \%$ to $29 \%$ in RA (data not shown).

In a multivariate analysis of the relation between BMD at different sites of measurement (dependent variables) and disease status (SLE, RA, healthy controls) as independent variables, the disease status of SLE appeared as an independent significant predictor of BMD at lumbar spine, when controlling for other independent variables (BMI, SF-36 physical, MHAQ, cumulative corticosteroid dose, smoking status and menopause in women). RA disease status had no independent predictive effect on BMD at any site of measurement (data not shown).

ASSOCIATION BETWEEN BMD, DEMOGRAPHIC AND DISEASE VARIABLES IN SLE

Results of bivariate relations of BMD at spine L2-L4 and femoral neck with continuous (Pearson) and categorical variables ( $t$ test) are shown in table 3. As expected, age and BMI correlated with BMD both at the lumbar spine and at the femoral neck (data for total hip are not shown as they appeared similar to femoral neck). Accumulated organ damage index score (SDI) correlated moderately with BMD at femoral neck $(r=-0.33)$ and weakly to BMD at lumbar spine $(r=-0.26)$. Menopause in the women was associated with a lower BMD at both lumbar spine and femoral neck $(\mathrm{p}<0.01$ to 0.03 ). BMD in current corticosteroid users were significantly reduced at lumbar spine $(\mathrm{p}=$ 0.04), but not at femoral neck. Current and cumulative corticosteroid doses correlated weakly to moderately with BMD, whereas duration on corticosteroids showed less correlation. Men had a significantly higher BMD at femoral neck compared with women, whereas no significant difference was found at lumbar spine. Disease duration, disease activity assessed by SLEDAI and history of smoking were not significantly related to the BMD values (table 3).

Linear regression analyses were performed to identify the most important variables predicting BMD at different sites of measurement in SLE. Eligible independent variables were demographic (age, sex, BMI, smoking), disease variables (disease duration, current and cumulative corticosteroid dose, SLEDAI, SDI). As disease activity, disease duration and smoking appeared not to be associated with BMD in univariate analyses these variables were excluded in the multivariate model. The results of the multivariate analyses are shown in table 4. Age, BMI and current corticosteroid dose were all independent significant predictors of BMD at the lumbar spine, and together explained $22 \%$ of the variance of $\mathrm{BMD}$, whereas BMD at femoral neck (table 4) and total hip (data not shown) was significantly predicted by age and BMI only.

\section{Discussion}

Our data demonstrate that the Norwegian SLE patients had significantly reduced BMD values at lumbar spine and hip compared with sex, age and geographical area matched healthy controls and similar values to that of sex, age and disease duration matched RA patients. The result indicates that SLE or its treatment have pervasive negative impact on BMD. Both trabecular (spine L2-L4) and mixed cortical and trabecular bone were affected causing generally reduced bone mass. Premenopausal women on corticosteroids were especially affected, whereas BMD values in patients never treated with corticosteroids approached matched healthy controls, indicating great impact of corticosteroid treatment on BMD in SLE. 
Our findings corroborate previous studies of SLE, showing significantly reduced bone mass at lumbar spine and hip hi-7 $910^{10}$ compared with healthy controls. In contrast with our study, Dhillon and coworkers ${ }^{8}$ did not find reduced BMD at lumbar spine L1-L4 in premenopausal SLE patients taking corticosteroids for six months or longer compared with healthy controls. Hansen and coworkers ${ }^{10}$ found reduced BMD at femoral neck and hand, but normal values at the spine and distal forearm, whereas $\mathrm{Li}$ et a $\bar{l}$ found BMD at femoral neck comparable to healthy controls. The conflicting results may be explained by different patient selection, as only premenopausal SLE patients with corticosteroid treatment were examined in some of the studies, ${ }^{3-579}$ limiting the value of direct comparison to ours.

The occurrence of OP in SLE was higher at all sites of measurement compared with matched healthy controls; the proportions comparing well with other prevalence studies of OP in white patients with $\mathrm{SLE}^{369}$ and was, as expected, higher than that of $4-6 \%$ in Chinese SLE patients. ${ }^{5}$ The proportion of patients with reduced bone mass was in line with that of an Australian study. ${ }^{6}$

Our study showed no significant differences in BMD values, frequencies of OP or reduced bone mass between matched SLE and RA patients at any site of measurement. The similar BMD values are interesting in view of the clearly documented impact of RA on BMD. ${ }^{24} 25$ The factors provoking reduced BMD may, however, be different in the two diseases. In $\mathrm{RA}$, reduced bone mass is mainly caused by the underlying inflammatory process, physical inactivity and corticosteroid treatment. ${ }^{26}{ }^{25}$ Physical disability is found to be less pronounced in SLE than in RA, ${ }^{27}$ because of milder inflammation in the joints, and hence less structural damages, which also is in accord with our findings (table1). SLE patients are more extensively exposed to corticosteroids compared with RA, as indicated by the present data (table 1). In SLE, factors other than corticosteroids such as avoidance of sun exposure, renal dysfunction, anticoagulation treatment (heparin or warfarin), ovarian dysfunction, premature menopause and avoidance of oestrogen replacement in women may also be important. ${ }^{28}$ In this study a small proportion of the SLE patients had renal disorder $(18 \%)$ or used anticoagulants (5\%). A higher proportion of the SLE patients was postmenopausal compared with the control groups, however, the use of oestrogen replacement therapy was similar in the three groups. The premature menopause in SLE patients could reflect the disease itself, its treatment or both. In addition, patients with SLE may not attain an optimum peak bone mass at skeletal maturity, as their disease often starts in early adult life. ${ }^{28}$ Finally, in both SLE and RA, studies suggest that inflammatory factors may change bone metabolism and contribute to the development of OP. ${ }^{29-32}$

Although similar BMD values in SLE and RA may confer similar risk of fractures, the SLE patients recorded somewhat higher proportion of previous osteoporotic fractures compared with RA (table1). Increased vertebral and hip fracture rates are known in $\mathrm{RA}^{33}$ and in women with SLE. ${ }^{34} 35$ Reduced BMD values are in general a strong risk factor for fractures in primary $\mathrm{OP},{ }^{36}{ }^{37}$ but it is not known if this is also the case in secondary OP. Indeed, the study of Peel et $a l^{33}$ of corticosteroid treated postmenopausal RA patients, showed that the decrease in lumbar spine BMD was less than expected in patients with fractures compared with those without. ${ }^{33}$ More studies are needed to clarify the role of BMD as predictor of later fracture in both RA and SLE.

Three previous studies have compared bone mass in SLE and RA, and showed conflicting results, however none of them used DEXA for measurement. ${ }^{12-14}$ In line with our study Dykman et $a l^{12}$ found no difference in proportions of glucocorticoid induced osteopenia and bone fractures in SLE and RA patients whereas Kalla et $a l^{13}{ }^{14}$ observed significantly reduced bone mass in RA patients only when performing radiometry in premenopausal SLE, RA and healthy controls. Kalla et $a l^{13}$ also observed more severe periosteal resorption by metacarpal measures in SLE, in contrast with endosteal resorption in RA, suggesting differences in the mechanism of osteoporosis in the two diseases.

The predictive role of age and BMI on BMD in SLE corroborates previous studies. As expected, current and cumulative corticosteroid doses correlated moderately with BMD at all sites of measurement. The results of the multivariate analysis confirmed that the current dose independently predicted BMD at lumbar spine, supporting that trabecular bone may be more sensitive to corticosteroids than cortical bone. ${ }^{38}$ The negative impact of corticosteroids on bone mass corroborates some studies $^{4610-1234}$ and contrasts others. ${ }^{3578}$ These discrepancies are unclear, but different practice in use of corticosteroids may be one explanation. ${ }^{69} \mathrm{BMD}$ values correlated better with accumulated damage (SDI) than with disease activity (SLEDAI) (table 3). Both $\mathrm{BMD}$ and organ damage capture cumulative changes over time, in contrast with disease activity recording new or deteriorating disease activity the last 10 days. The lack of correlation between BMD, disease duration and disease activity agrees with previous studies. ${ }^{368-11}$

Limitations of this study are the relatively small patient samples, lack of data on biochemical and hormonal parameters relevant to reduced bone mass in SLE and RA, lack of analysis of spinal deformities and lack of data using hand as a possible site of measurement. Data on structural damage-that is, radiographic abnormalities in RA patients was not available. Strength of our study is the successful demographic matches of the study populations, permitting a case-control design. When matching SLE and RA patients, we controlled for two independent and important variables, namely age and disease duration. The higher proportion of SLE patients with menopause compared with the control groups may, however, skew the BMD data, as menopause has an important impact on BMD. The data of RA patients included from the register are 
reasonably representative for the RA population in Oslo, ${ }^{16}$ and their BMD concurred with that in RA patients aged 40-49 years in that register. The low coefficient of variation of the DEXA data assured that standard procedures were followed. The in vivo reproducibility of BMD measurements of duplicate measurements in 31 healthy female hospital workers was also satisfactory.

To conclude, the study showed that our SLE patients had reduced bone mass at all sites of measurement compared with age and sex matched healthy controls. Especially the premenopausal women taking corticosteroids were affected, whereas patients with mild SLE and no corticosteroid use had bone mass values approaching the normal population. Furthermore, the bone mass of matched patients with SLE and RA was reduced to a similar extent. The study also showed that the SLE patients received more corticosteroids than the RA patients and underwent menopause at an earlier age whereas the RA patients had more severe physical disability. Disease activity and duration did not affect BMD in SLE, whereas corticosteroids did. Clinical implications of this study include emphasis on lowering corticosteroid dose if possible, and prevention and treatment of OP in SLE patients taking corticosteroids.

The authors gratefully appreciate our technicians Ingerid Müller, Sidsel Arnkværn and Espen Haavardsholm for expert technical assistance and Dr Till Uhlig providing clinical data on the RA patients.

Funding: the study is in part supported by grants from the Lions Clubs International MD 104 Norway, the Norwegian Rheumatism Association, the Norwegian Research Council, the Norwegian Women's Public Health Association, the Legacy of Grethe Harbitz and the Legacy of Trygve Gythfeldt and Wife.

1 Gladman D, Ginzler E, Goldsmith C, Fortin P, Liang M, Urowitz M, et al. The development and initial validation of
the Systemic Lupus International Collaborating Clinics/ the Systemic Lupus International Collaborating Clinics/
American College of Rheumatology damage index for sysAmerican College of Rheumatology damage index for sys-
temic lupus erythematosus. Arthritis Rheum temic lupus

2 Anonymous. Consensus Development Conference: Prophylaxis and treatment of Osteoporosis. Osteoporosis In 1991;114-17

3 Pons F, Peris P, Guanabens N, Font J, Huguet M, Espinosa $\mathrm{G}$, et al. The effect of systemic lupus erythematosus and long-term steroid therapy on bone mass in pre-menopausal women. Br J Rheumatol 1995; 34:742-6.

4 Houssiau FA, Lefebvre C, Depresseux G, Lambert M, Devogelaer J-P, Nagant de Deuxchaines C. Trabecular and cortical bone loss in systemic lupus erythematosus. Br J Rheumatol 1996;35:244-7.

5 Li EK, Tam LS, Young RP, Ko GTC, Li M, Lau EMC. Loss of bone mineral density in Chinese pre-menopausal women of bone mineral density in Chinese pre-menopausal women with systemic lupus erythematosus treated

6 Kipen Y, Buchbinder R, Forbes A, Strauss B, Littlejohn G, Morand E. Prevalence of reduced bone mineral density in systemic lupus erythematosus. J Rheumatol 1997; 24 1922-9.

7 Kalla AA, Fataar AB, Jessop S, Bewerunge L. Loss of trabecular bone mineral density in systemic lupus erythematosus. Arthritis Rheum 1993;36:1726-34

8 Dhillon VB, Davies M, Hall ML, Round J, Ell PJ, Jacobs HS, et al. Assessment of the effect of oral corticosteroids on bone mineral density in systemic lupus erythematosus: A preliminar study with dual enery $\mathrm{x}$ - ray absorptiometry. Ann Rheum Dis 1990; 49:624-6.

9 Formiga F, Moga I, Nolla JM, Pac M, Mitjavila F, Roig-Escofet D. Loss of bone mineral density in premenopausal women with systemic lupus erythematosus. Ann Rheum Dis 1995;54:274-6.

10 Hansen M, Halberg P, Kollerup G, Pedersen-Zbinden B, Horslev-Petersen K, Hyldstrup L, et al. Bone metabolism in patients with systemic lupus erythematosus. Effect of disease activity and glycocorticoid treatment. Scand J disease activity and glycocortic
Rheumatol 1998; 27:197-206.
11 Kipen Y, Briganti E, Strauss B, Will R, Littlejohn G, Morand E. Three years follow-up of bone mineral density change in premenopausal women with systemic lupus erythematosus. J Rheumatol 1999;26:310-17.

12 Dykman TR, Gluck OS, Murphy W, Hahn T, Hahn BH. Evaluation of factors associated with glucocorticoidinduced osteopenia in patients with rheumatic diseases. Arthritis Rheum 1985;28:361-8.

13 Kalla AA, Meyers OL, Parkyn N, Kotze T. Osteoporosis screening-radiogrammetry revisited. Br J Rheumatol 1989; 28:511-17.

14 Kalla AA, Meyers OL, Kotze T, Laubesher R. Corticosteroid therapy and bone mass - comparison of rheumatoid
arthritis and systemic lupus erythematosus. S Afr Med J arthritis and sys

15 Kvien TK, Glennås A, Knudsrød OG, Smedstad LM, Mowinkel P, Førre Ø. The prevalence and severity of rheumatoid arthritis in Oslo: Result form a county register and a population survey. Scand J Rheumatol 1997;26:412-18.

16 Uhlig T, Kvien TK, Glennås A, Smedstad LM, Førre Ø. The incidence and severity of rheumatoid arthritis. Results from a county register in Oslo, Norway. J Rheumatol 1998; 25:1078-84.

17 Tan EM, Cohen AS, Fries JF, Alfonse TM, McShane DJ, Rothefield NF, et al. The 1982 revised criteria for the classification of systemic lupus erythematosus. Arthritis Rheum 1982;25:1271-7.

18 Arnett FC, Edworthy SM, Bloch AB, McShane DJ, Fries JF, Cooper NS. The American Rheumatism Association 1987 revised criteria for the classification of rheumatoid artritis. Arthritis Rheum 1988;31:315-24.

19 Pincus T, Callahan LF, Brooks RH, Fuchs HA, Olsen NJ, Kaye JJ. Assessment of patient satisfaction in activities of daily living using a modified Stanford health assessment questionnaire. Arthritis Rheum 1983;26:46-53.

20 Ware JE, Sherbourne CD. The MOS 36-item Short Form health Survey (SF-36) I. Conceptual framework and item selection. Med Care 1992;30:473-83.

21 Fries JF, Spitz PW, Kraines RG, Holman HR. Measurement of patient outcome in arthritis. Arthritis Rheum 1980;23: $37-45$.

22 Bombardier C, Gladman DD, Urowitz MB, Caron D, Chang $\mathrm{CH}$, and the committee on prognosis studies in SLE. Derivation of SLEDAI. Arthritis Rheum 1992;35: 30-40.

23 Kanis JA, Melton LJ, Christiansen C, Johnston CC, Khaltaev N. The diagnosis of osteoporosis. J Bone Miner Kes $1994 \cdot 9: 1137-41$.

24 Deodhar AA, Woolf AD. Bone mass measurement and bone metabolism in rheumatoid arthritis: A review. $\mathrm{Br} \mathrm{J}$ Rheumatol 1996;35:309-22.

25 Hall GM, Spector TD, Griffin AJ, Jawad AS, Hall ML, Doyle DV. The effect of rheumatoid arthritis and steroid therapy on bone density in postmenopausal women. Arthritis Rheum 1993;36:1510-16.

26 Martin JC, Munro R, Campbell MK, Reid DM. Effects of disease and corticosteroids on appendicular bone mass in postmenopausal women with rheumatoid arthritis: comparison with axial measurements. Br J Rheumatol 1997;36: 43-9.

27 Gilboe IM, Kvien TK, Husby G. Health status in systemic lupus erythematosus compared to rheumatoid arthritis and lupus erythematosus compared to rheumatoid arth

28 Segal LG, Lane NE. Osteoporosis and systemic lupus erythematosus: etiology and treatment strategies. Ann Med Interne (Paris) 1996;147:281-9.

29 McLeod JF. Osteoporosis, cytokines, and glucocorticoids. Allergy Proc 1993;14:363-4

30 Tanaka Y, Watanabe K, Suzuki M, Saito K, Oda S, Suzuki $\mathrm{H}$, et al. Spontaneous production of bone-resorbing lymphokines by B cells in patients with systemic lupus erythematosus. J Clin Immunol 1989;9:415-20.

31 Seitz M, Hunstein W. Enhanced prostanoid release from monocytes of patients with rheumatoid arthritis and active systemic lupus erythematosus. Ann Rheum Dis 1985;44: 438-45.

32 Maury C, Teppo A-M. Tumor necrosis factor in the serum of patients with systemic lupus erythematosus. Arthritis of patients with system

33 Peel NF, Moore DJ, Barrington NA, Bax DE, Eastell R. Risk of vertebral fracture and relationship to bone mineral density in steroid treated rheumatoid arthritis. Ann Rheum Dis 1995;54:801-6.

34 Petri M. Musculoskeletal complications of systemic lupus erythematosus in the Hopkins Lupus Cohort: An update. Arthritis Care Res 1995;8:37-45.

35 Ramsey-Goldman R, Dunn J, Huang C-F, Dunlop D, Rairie $\mathrm{J}$, Fritzgerald $\mathrm{S}$, et al. Frequency of fractures in women with systemic lupus erythematosus. Arthritis Rheum 1999;42: 882-90.

36 Ross PD, Davis JW, Epstein RS, Wasnich RD. Pre-existing fractures and bone mass predict vertebral fracture incidence in women. Ann Intern Med 1991;114:919-23.

37 Cummings SR, Black DM, Nevitt MC, Browner W, Cauley $\mathrm{J}$, Ensrud $\mathrm{K}$, et al. Bone density at various sites for prediction of hip fractures. The Study of Osteoporotic Fractures Research Group. Lancet 1993;341:72-5.

38 Sambrook PN, Jones G. Corticosteroid osteoporosis. Br J Rheumatol 1995;34:8-12. 